\title{
WEAK CONVERGENCE OF SEMIGROUPS IMPLIES STRONG CONVERGENCE OF WEIGHTED AVERAGES
}

\author{
HUMPHREY FONG
}

\begin{abstract}
For a fixed $p, 1 \leqslant p<\infty$, let $\left\{T_{t}: t>0\right\}$ be a strongly continuous semigroup of positive contractions on $L_{p}$ of a $\sigma$-finite measure space. We show that weak convergence of $\left\{T_{t}: t>0\right\}$ in $L_{p}$ is equivalent with the strong convergence of the weighted averages $\int_{0}^{\infty} T_{t} f \mu_{n}(d t)(n \rightarrow \infty)$ for every $f \in L_{p}$ and every sequence $\left(\mu_{n}\right)$ of signed measures on $(0, \infty)$, satisfying $\sup _{n}\left\|\mu_{n}\right\|<\infty ; \lim _{n} \mu_{n}(0, \infty)=1$; and for each $d>0, \lim _{n} \sup _{c>0}\left|\mu_{n}\right|(c, c$ $+d]=0$. The positivity assumption is not needed if $p=1$ or 2 . We show that such a result can be deduced-not only in $L_{p}$, but in general Banach spaces-from the corresponding discrete parameter version of the theorem.
\end{abstract}

In recent years, various authors have studied the relations between weak and strong operator convergence: Blum-Hanson [4], Hanson-Pledger [7], Lin [9], Akcoglu-Sucheston [1], Jones-Kuftinec [8], Fong-Sucheston [6], and very recently Akcoglu-Sucheston [2], [3], who proved the theorem for positive $L_{p^{-}}$ contractions, $1<p<\infty$. This theorem [1], [6], [3] states that if $T$ is a positive contraction on $L_{p}$ of a $\sigma$-finite measure space $(X, \mathbb{Q}, m)$, where $p$ is fixed and $1 \leqslant p<\infty$, then weak- $\lim _{n \rightarrow \infty} T^{n} f\left(\mathrm{w}-\lim _{n} T^{n} f\right)$ exists for each $f \in L_{p}$ if, and only if, $\lim _{n \rightarrow \infty} \sum_{m=1}^{\infty} a_{n m} T^{m} f$ exists for every $f \in L_{p}$ and every matrix $\left(a_{n m}\right)$ with real entries satisfying

$$
\sup _{n} \sum_{m}\left|a_{n m}\right|<\infty ; \lim _{n} \sum_{m} a_{n m}=1 ; \lim _{n} \max _{m}\left|a_{n m}\right|=0 .
$$

It has also been shown that the positivity assumption is not needed if $p=1$ or 2 [1], [6]. The problem of whether or not positivity is needed for $p \neq 1$ or 2 is still open. Matrices satisfying (1.1) were introduced in ergodic context in [6] and have been called uniformly regular; we denote the class of all uniformly regular matrices by $\mathfrak{A}_{R}$. Intuitively, a matrix $\left(a_{n m}\right) \in \mathfrak{A}_{R}$ if and only if it is properly averaging, in the sense that the masses $a_{n m}$ spread as $n \rightarrow \infty$.

A semigroup $\left\{T_{t}: t>0\right\}, T_{t} T_{s}=T_{t+s}$, of linear operators on a Banach space $B$ is called strongly continuous if for each $x \in B$ and each $s>0$, $\lim _{t \rightarrow s}\left\|T_{t} x-T_{s} x\right\|=0$. R. Sato [10] recently obtained the following continuous parameter version of the strong ergodic theorem: For a fixed function $f$ in $L_{2}$ and a strongly continuous semigroup $\left\{T_{t}: t>0\right\}$ of contractions on

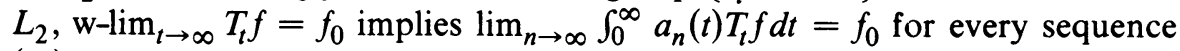
$\left(a_{n}\right)$ of nonnegative, Lebesgue integrable functions on $(0, \infty)$ satisfying

Received by the editors January 3, 1975 and, in revised form, April 9, 1975.

AMS (MOS) subject classifications (1970). Primary 47A35; Secondary 28A45.

Key words and phrases. Banach space, $L_{p}$-space, semigroup of operators, weak convergence, ergodic theorem. 
$\int_{0}^{\infty} a_{n}(t)=1$ and $\lim _{n \rightarrow \infty}\left\|a_{n}\right\|_{\infty}=0$. In this note, we show that a stronger result can be deduced-not only in $L_{2}$, but in general Banach spaces-from the corresponding discrete version of the theorem (\$2). In \$3, we obtain as corollaries the continuous parameter version of the Akcoglu-Sucheston theorem for the Banach spaces $L_{p}(X, \mathbb{Q}, m), 1 \leqslant p<\infty$.

2. A linear operator $T$ on a Banach space $B$ is called power-bounded if $\sup _{n}\left\|T^{n}\right\|<\infty$; a semigroup $\left\{T_{t}: t>0\right\}$ of linear operators on $B$ is called uniformly bounded if $\sup _{t>0}\left\|T_{t}\right\|<\infty$. The total variation of a signed measure $\mu$ is denoted by $|\mu|$. We denote by $\mathfrak{A}$ the family of all sequences $\left(\mu_{n}\right)$ of signed measures on the $\sigma$-algebra of Lebesgue measurable subsets of $(0, \infty)$ satisfying

$$
\begin{aligned}
& \sup _{n}\left\|\mu_{n}\right\|<\infty ; \lim _{n} \mu_{n}(0, \infty)=1 ; \\
& \lim _{n \rightarrow \infty} \sup _{c \geqslant 0}\left|\mu_{n}\right|(c, c+d]=0 \quad \text { for each } d>0 .
\end{aligned}
$$

REMARK 1. If $\left(a_{n}\right)$ is a sequence of Lebesgue integrable functions on $(0, \infty)$ satisfying

$$
\begin{gathered}
\sup _{n} \int_{0}^{\infty}\left|a_{n}(t)\right| d t<\infty ; \quad \lim _{n} \int_{0}^{\infty} a_{n}(t) d t=1 ; \\
\lim _{n \rightarrow \infty} \sup _{c \geqslant 0} \int_{c}^{c+d}\left|a_{n}(t)\right| d t=0
\end{gathered}
$$

for each $d>0$, and if we set $d \mu_{n}=a_{n} d t$, then $\left(\mu_{n}\right) \in \mathfrak{A}$. We note that sequences $\left(a_{n}\right)$ satisfying (2.2) include those considered by Sato in [10].

REMARK 2. Let $\delta(t)$ denote the unit point mass at $t$. If $t_{m}>0, t_{m} \rightarrow \infty$, $\left(a_{n m}\right) \in \mathfrak{A}_{R}$, and if we set $\mu_{n}=\sum_{m} a_{n m} \delta\left(t_{m}\right)$, then $\left(\mu_{n}\right) \in \mathfrak{U}$.

REMARK 3. If $x(t)$ is a bounded continuous function from $(0, \infty)$ to a Banach and if we set $d \mu_{n}=a_{n} d t$, then $\left(\mu_{n}\right) \in \mathfrak{A}$. We note that sequences $\left(a_{n}\right)$ satisfying (2.2) include those considered by Sato in [10].

$$
\left\|\int_{0}^{\infty} x(t) \mu(d t)\right\| \leqslant\left(\sup _{t \geqslant 0}\|x(t)\|\right) \cdot\|\mu\| \quad \text { (cf. [5]). }
$$

THEOREM 2.1. Let $x$ be a fixed element in a Banach space $B$, real or complex. Then $(\alpha)$ implies $(\beta)$ :

$(\alpha)$ For every power bounded linear operator $T$ on $B$, if $\mathrm{w}-\lim _{n \rightarrow \infty} T^{n} x=x_{0}$, then $\lim _{n \rightarrow \infty} \sum_{m=1}^{\infty} a_{n m} T^{m} x=x_{0}$ for every matrix $\left(a_{n m}\right) \in \mathfrak{A}_{R}$.

$(\beta)$ For every uniformly bounded semigroup $\left\{T_{t}: t>0\right\}$ of linear operators on $B$ for which $T_{t} x$ is continuous on $(0, \infty)$, if $\mathrm{w}-\lim _{t \rightarrow \infty} T_{t} x=x_{0}$, then $\lim _{n} \int_{0}^{\infty} T_{t} x \mu_{n}(d t)=x_{0}$ for every sequence $\left(\mu_{n}\right) \in \mathfrak{A}$.

The conclusion remains valid if "power bounded" and "uniformly bounded" in $(\alpha)$ and $(\beta)$ are both replaced by "contraction".

Proof. Let $x$ be a fixed element in $B$, and assume that $(\alpha)$ holds for $x$. Let $\left\{T_{t}: t>0\right\}$ be a semigroup satisfying the hypotheses of $(\beta)$ and $\left(\mu_{n}\right) \in \mathfrak{A}$. We shall show that $\lim _{n} \int_{0}^{\infty} T_{t} x \mu_{n}(d t)=x_{0}$.

Let $\varepsilon>0$. The continuity of $T_{t} x$ on $[1,2]$ implies that $T_{t} x$ is uniformly continuous on $[1,2]$. Thus there is a positive integer $k$ such that if 


$$
g(t)=\sum_{j=1}^{k} 1_{(1+(j-1) / k, 1+j / k)}(t) \cdot T_{1+j / k} x,
$$

then $\left\|g(t)-T_{t} x\right\|<\varepsilon$ for $t \in(1,2]$. Here $1_{A}$ denotes the function that is 1 on $A$, and 0 elsewhere. Set $M=\sup _{t>0}\left\|T_{t}\right\|, K=\sup _{n}\left\|\mu_{n}\right\|$, and $I_{i}=(i, i+1]$. Since $\left|\mu_{n}\right|\left(I_{0}\right) \rightarrow 0$ by $(2.1)$, we have

$$
\begin{aligned}
\underset{n \rightarrow \infty}{\limsup } \| & \left\|\int_{0}^{\infty} T_{t} x \mu_{n}(d t)-\sum_{i=0}^{\infty} \int_{I_{i+1}} T_{i} g(t-i) \mu_{n}(d t)\right\| \\
& =\limsup _{n \rightarrow \infty}\left\|\int_{I_{0}} T_{t} x \mu_{n}(d t)+\sum_{i=0}^{\infty} \int_{I_{i+1}}\left(T_{t} x-T_{i} g(t-i)\right) \mu_{n}(d t)\right\| \\
& \leqslant \limsup _{n \rightarrow \infty}\left[M \cdot\|x\| \cdot\left|\mu_{n}\right|\left(I_{0}\right)\right. \\
& \left.+\sum_{i=0}^{\infty}\left\|T_{i}\right\| \cdot \sup _{t \in I_{1}}\left\|T_{t} x-g(t)\right\| \cdot\left|\mu_{n}\right|\left(I_{i+1}\right)\right] \\
\leqslant &
\end{aligned}
$$

For each $i \geqslant 0,1 \leqslant j \leqslant k$, set $I_{i, j}=(i+(j-1) / k, i+j / k$ ]. It follows from the definition of $g(t)$ that for $n \geqslant 1$,

$$
\begin{aligned}
\sum_{i=0}^{\infty} \int_{I_{i+1}} T_{i} g(t-i) \mu_{n}(d t) & =\sum_{i=0}^{\infty} \sum_{j=1}^{k} \mu_{n}\left(I_{i+1, j}\right) \cdot T_{i+1+j / k} x \\
& =\sum_{m=k+1}^{\infty} a_{n, m} T^{m} x,
\end{aligned}
$$

where $\quad T=T_{1 / k}$, and for $m=(i+1) k+j, i \geqslant 0,1 \leqslant j \leqslant k, a_{n, m}$ $=\mu_{n}\left(I_{i+1, j}\right)$. It is easily checked that $\left(a_{n, m}\right) \in \mathfrak{U}_{R}$ since $\left(\mu_{n}\right) \in \mathfrak{A}$. Moreover,

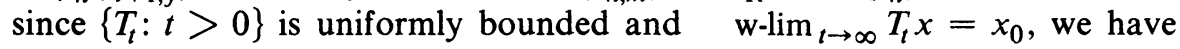
that $T$ is power bounded and $\quad w-\lim _{m \rightarrow \infty} T^{m} x=x_{0}$. Thus it follows from ( $\alpha$ ) that $\lim _{n \rightarrow \infty} \sum_{m>k} a_{n m} T^{m} x=x_{0}$. Together with (2.3) and (2.4), we obtain that

$$
\limsup _{n \rightarrow \infty}\left\|\int_{0}^{\infty} T_{t} x \mu_{n}(d t)-x_{0}\right\| \leqslant M K \varepsilon .
$$

As $\varepsilon>0$ is arbitrary, $(\beta)$ holds.

It is clear that the second part of the theorem can be proved in the same way.

Corollary 2.1. Let $B$ be a Banach space. Then $(\alpha)^{\prime}$ implies $(\beta)^{\prime}$ :

$(\alpha)^{\prime}$ For every power bounded linear operator $T$ on $B$, if $\mathrm{w}-\lim _{n \rightarrow \infty} T^{n} x$ exists for every $x \in B$, then $\lim _{n \rightarrow \infty} \sum_{m=1}^{\infty} a_{n, m} T^{m} x$ exists and is equal to w- $\lim _{n \rightarrow \infty} T^{n} x$ for every $\left(a_{n, m}\right) \in \mathfrak{A}_{R}$.

$(\beta)^{\prime}$ For every strongly continuous, uniformly bounded semigroup $\left\{T_{t}: t>0\right\}$ of linear operators on $B$, if $\mathrm{w}-\lim _{t \rightarrow \infty} T_{t} x$ exists for every $x \in B$, then $\lim _{n} \int_{0}^{\infty} T_{t} x \mu_{n}(d t)$ exists for every sequence $\left(\mu_{n}\right) \in ! \mathfrak{A}$, and is equal to $\mathrm{w}-\lim _{t \rightarrow \infty} T_{t} x$.

The conclusion remains valid if "power bounded" and "uniformly bounded" are both replaced by "contraction". 
Proof. Immediate from Theorem 2.1 .

We next show that the converse of statement $(\beta)$ in Theorem 2.1 is valid in general Banach spaces.

Proposition 2.1. Let $x$ be a fixed element in a Banach space $B$, real or complex. Let $\left\{T_{t}: t>0\right\}$ be continuous linear operators on $B$ such that the vectorvalued function $T_{(\cdot)} x$ from $(0, \infty)$ to $B$ is continuous and $\sup _{t>0}\left\|T_{t} x\right\|<\infty$. Then (b) implies (a):

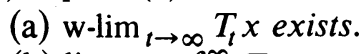

(b) $\lim _{n \rightarrow \infty} \int_{0}^{\infty} T_{t} x \mu_{n(d t)}$ exists for every sequence $\left(\mu_{n}\right) \in \mathfrak{U}$.

Proof. We first consider the case where $B$ is a real Banach space. Assume that (b) holds but (a) fails. Then there exists an $x^{*} \in B^{*}$ such that $h(t)$ $=\left\langle T_{t} x, x^{*}\right\rangle$ diverges as $t \rightarrow \infty$, where $B^{*}$ is the dual space of $B$. Since

$$
\sup _{t>0}|h(t)| \leqslant \sup _{t>0}\left\|x^{*}\right\|\left\|T_{t} x\right\|<\infty
$$

$h$ is bounded on $(0, \infty) . h(t)$ is also continuous on $(0, \infty)$ since $T_{t} x$ is. Thus, $h(t)$ being divergent as $t \rightarrow \infty$, there are constants $\alpha, \beta$ with $\alpha<\beta$, and a sequence $\left(t_{i}\right)$ with $t_{i} \uparrow \infty$, such that $h\left(t_{i}\right) \geqslant \beta$ if $i$ is odd, and $h\left(t_{i}\right) \leqslant \alpha$ if $i$ is even. Set for $n \geqslant 1$,

$$
\mu_{2 n}=\frac{1}{n} \sum_{k=1}^{n} \delta\left(t_{2 k}\right), \quad \mu_{2 n-1}=\frac{1}{n} \sum_{k=1}^{n} \delta\left(t_{2 k-1}\right),
$$

where $\delta(t)$ denotes the unit point mass at $t$. Then $\left(\mu_{n}\right) \in \mathfrak{A}$, but

$$
\begin{aligned}
\liminf _{n}\left\langle\int_{0}^{\infty}\right. & \left.T_{t} x \mu_{n}(d t), x^{*}\right\rangle=\liminf _{n \rightarrow \infty} \frac{1}{n} \sum_{k=1}^{n} h\left(t_{2 k}\right) \\
& \leqslant \alpha<\beta \leqslant \limsup \frac{1}{n} \sum_{k=1}^{n} h\left(t_{2 k-1}\right) \\
& =\limsup _{n \rightarrow \infty}\left\langle\int_{0}^{\infty} T_{t} x \mu_{n}(d t), x^{*}\right\rangle .
\end{aligned}
$$

Hence $\left(\int_{0}^{\infty} T_{t} x \mu_{n}(d t)\right)_{n=1}^{\infty}$ does not converge weakly, and a fortiori, strongly.

If $B$ is a complex Banach space, then either the real part or the imaginary part of $h(t)$ diverges as $t \rightarrow \infty$, and can be used to replace $h(t)$ in the above argument.

Remark 4. The vector-valued function $T_{t} x$ in Proposition 3.1 may be replaced by any vector-valued function $x(t)$ from $(0, \infty)$ to $B$ such that $x(t)$ is continuous and bounded on $(0, \infty)$.

3. We now apply the results in $\S 2$ to the Banach spaces $L_{p}$ of a $\sigma$-finite measure space $(X, Q, m), 1 \leqslant p<\infty$. An operator $T$ on $L_{p}$ is called positive if $T f \geqslant 0$ whenever $f \geqslant 0$. Theorem 3.1 below strengthens the result of $\mathbf{R}$. Sato mentioned in $\$ 1$.

THEOREM 3.1. Let $\left\{T_{t}: t>0\right\}$ be a contraction semigroup on $L_{2}(X, \mathbb{Q}, m)$, and let $f$ be a fixed function in $L_{2}$ such that $T_{f} f$ is continuous on $(0, \infty)$. Then conditions (a) and (b) are equivalent: 
(a) $\mathrm{w}-\lim _{t \rightarrow \infty} T_{t} f=f_{0}$.

(b) $\lim _{n} \int_{0}^{\infty} T_{t} f \mu_{n}(d t)=f_{0}$ for every $\left(\mu_{n}\right) \in \mathfrak{A}$.

Proof. This follows from Theorem 1.1 in [6], Proposition 2.1 and Theorem 2.1.

THEOREM 3.2. Let $\left\{T_{t}: t>0\right\}$ be a strongly continuous contraction semigroup on $L_{1}(S, \mathbb{Q}, m)$. Then conditions $(\mathrm{A})$ and $(\mathrm{B})$ are equivalent:

(A) For each $f \in L_{1}$, w- $\lim _{t \rightarrow \infty} T_{t} f$ exists.

(B) For each $f \in L_{1}, \lim _{n \rightarrow \infty} \int_{0}^{\infty} T_{t} f \mu_{n}(d t)$ exists for every $\left(\mu_{n}\right) \in \mathfrak{U}$, and is equal to $\mathrm{w}-\lim _{t \rightarrow \infty} T_{t} f$.

Proof. This follows from Theorem 1.3 in [6], Proposition 2.1, and Corollary 2.1.

THEOREM 3.3 Let $\left\{T_{t}: t>0\right\}$ be a strongly continuous semigroup of positive contractions on $L_{p}(X, \mathbb{Q}, m)$, where $p$ is fixed, $1<p<\infty$. Then conditions (A) and $(\mathrm{B})$ are equivalent:

(A) For each $f \in L_{p}$, w- $\lim _{t \rightarrow \infty} T_{t} f$ exists.

(B) For each $f \in L_{p}, \lim _{n \rightarrow \infty} \int_{0}^{\infty} T_{t} f \mu_{n}(d t)$ exists for each sequence $\left(\mu_{n}\right) \in \mathfrak{U}$, and is equal to $\mathrm{w}-\lim _{n \rightarrow \infty} T_{t} f$.

Proof. We observe that the conclusions in Theorem 2.1 and Corollary 2.1 remain valid if $B=L_{p}(X, Q, m)$, and "power bounded" and "uniformly bounded" in $(\alpha)$ and $(\beta)$ are both replaced by "positive contraction". Theorem 3.3 now follows from Theorem 1.4 in [3] and Proposition 2.1.

Acknowledgement. The author wishes to thank Professor M. Lin for valuable comments, and Professors Akcoglu and Sucheston for making available to him their papers [2] and [3] prior to publication.

\section{REFERENCES}

1. M. Akcoglu and L. Sucheston, On operator convergence in Hilbert space and in Lebesgue space, Period. Math. Hungar. 2 (1972), 235-244. MR 48 \# 4777.

2. - On convergence of iterates of positive contractions in $L_{p}$ spaces, J. Approximation Theory (to appear).

3. - On weak and strong convergence of positive contractions in $L_{p}$ spaces, Bull. Amer. Math. Soc. 81 (1975), 105-106.

4. J. R. Blum and D. L. Hanson, On the mean ergodic theorem for subsequences, Bull. Amer. Math. Soc. 66 (1960), 308-311. MR 22 \#9572.

5. N. Dunford and J. T. Schwartz, Linear operators. I: General theory, Pure and Appl. Math., vol. 7, Interscience, New York, 1958. MR 22 \#302.

6. H. Fong and L. Sucheston, On a mixing property of operators in $L_{p}$ spaces, $Z$. Wahrscheinlichkeitstheorie und Verw. Gebiete 28 (1974), 165-171.

7. D. L. Hanson and G. Pledger, On the mean ergodic theorem for weighted averages, $\mathrm{Z}$. Wahrscheinlichkeitstheorie und Verw. Gebiete 13 (1969), 141-149. MR 40 \#423.

8. L. K. Jones and V. Kuftinec, A note on the Blum-Hanson theorem, Proc. Amer. Math. Soc. 30 (1971), 202-203. MR 43 \#6742.

9. M. Lin, Mixing for Markov operators, Z. Wahrscheinlichkeitstheorie und Verw. Gebiete 19 (1971), 231-242. MR 46 \#8317.

10. R. Sato, A note on operator convergence for semi-groups, Comment. Math. Univ. Carolinae 15 (1974), 127-129.

Department of Mathematics, Bowling Green State University, Bowling Green, Ohio 43403 